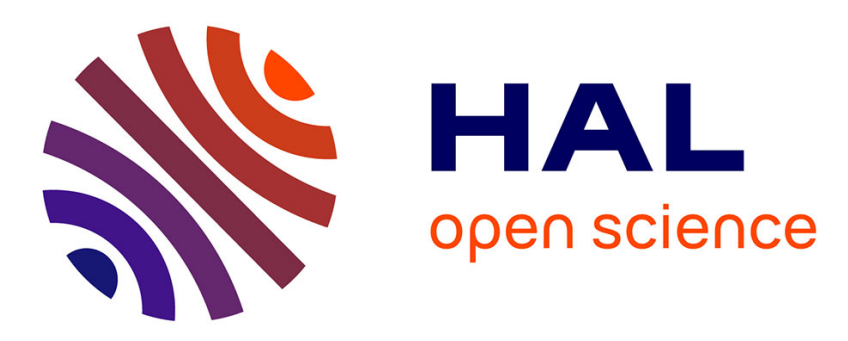

\title{
Eye dominance modulates visuospatial attention
}

S Schintu, R Chaumillon, A Guillaume, R Salemme, K T Reilly, L Pisella, A

Farnè

\section{To cite this version:}

S Schintu, R Chaumillon, A Guillaume, $\mathrm{R}$ Salemme, $\mathrm{K} \mathrm{T}$ Reilly, et al.. Eye dominance modulates visuospatial attention. Neuropsychologia, 2020, 141, pp.107314. 10.1016/j.neuropsychologia.2019.107314 . hal-03023110

\section{HAL Id: hal-03023110 https://hal.science/hal-03023110}

Submitted on 25 Nov 2020

HAL is a multi-disciplinary open access archive for the deposit and dissemination of scientific research documents, whether they are published or not. The documents may come from teaching and research institutions in France or abroad, or from public or private research centers.
L'archive ouverte pluridisciplinaire HAL, est destinée au dépôt et à la diffusion de documents scientifiques de niveau recherche, publiés ou non, émanant des établissements d'enseignement et de recherche français ou étrangers, des laboratoires publics ou privés. 


\title{
Eye dominance modulates visuospatial attention S. Schintu a,b,c,d, ${ }^{*}$, R. Chaumillon e, A. Guillaume f, R. Salemme a,b,g, K.T. Reilly a,b, L. Pisella a,b, A. Farnè $a, b, g, h$
}

\author{
a Integrative Multisensory Perception Action \& Cognition Team (ImpAct), INSERM U1028, CNRS UMR5292, Lyon Neuroscience Research Center (CRNL), \\ Lyon, France \\ b University of Lyon 1, Lyon, France \\ c Department of Psychology, George Washington University, WA, USA \\ d Behavioral Neurology Unit, National Institute of Neurological Disorders and Stroke, National Institutes of Health, Bethesda, MD, USA \\ e Aix Marseille Université, CNRS, LNC UMR 7291, FR 3C FR 3512, Marseille, France \\ f Paris Descartes University, Vision Action Cognition Laboratory, EA7326, Institute of Psychology, Boulogne-Billancourt, Paris, France \\ g Hospices Civils de Lyon, Mouvement \& Handicap, Neuro-Immersion Platforms, Lyon, France \\ $\mathrm{h}$ Center for Mind/Brain Sciences (CIMeC), University of Trento, Italy
}

\section{Keywords:}

Pseudoneglect

Asymmetry

Laterality

Visual cortex

Line bisection

\section{A B S TRACT}

Visuospatial attention has an inherent asymmetry: the leftward bias called pseudoneglect. In typical line bisection tasks, healthy individuals tend to judge the center of a line leftward of the true center, an effect attributed to the right hemisphere dominance in visuospatial attention. Since it has been shown that information perceived by the dominant eye strongly activates the ipsilateral visual cortex, we hypothesized that eye dominance may modulate visuospatial attention bias. Because activation of the left hemisphere induced by left eye dominance should mitigate the right hemisphere dominance in attention, we predicted that right-handed individuals with left dominant eye would show smaller amount of pseudoneglect than right-handed individuals with right dominant eye. We compared the performance at both the perceptual (Landmark) and manual line bisection task of forty right-handed healthy individuals, half of whom had a right dominant eye and the other half a left dominant eye. As predicted, the left eyed dominant group showed smaller, actually not significant pseudoneglect, which was thus greater in the right eye dominant group. The influence of eye dominance on visuospatial attention was present in the Landmark but not the manual line bisection task, in which the amount of visuospatial bias correlated with participants' degree of (right) handedness. This is the first report of the effect of eye dominance on visuospatial attention within a right-handed population. This finding, by showing the influence of eye dominance on visuospatial cognition, not only helps in better defining intact visuospatial cognition mechanism but also encourages further research to pinpoint the neural basis of such interaction.

\section{Introduction}

Young individuals free of neurological disorders usually judge the center of a line leftward of the true center as measured by line bisection tasks (Milner et al., 1992; Schenkenberg et al., 1980). This leftward bias in intact visuospatial attention is called pseudoneglect (Bowers and Heilman, 1980; for a review see Jewell and McCourt, 2000). Pseudoneglect is hypothesized to be the product of the uneven distribution of attention due to the right hemispheric dominance in attention (Kinsbourne, 1970; Mesulam, 1999; Nicholls and Roberts, 2002). The right hemisphere is indeed the most activated one during attentional task and, as the activation-orientation theory posits, this increased attention to the contralateral (left) hemifield makes the left visual space (i.e., left side of the line) being perceived longer then the right one, and thus judging the center of line leftward of the true center (Bultitude and Aimola Davies, 2006). Pseudoneglect is typically present at the population level, but much inter individual variability is observed (Manning et al., 1990; McCourt and Olafson, 1997; Jewell and McCourt, 2000; Toba et al., 2011; Szczepanski and Kastner, 2013). Among the several factors influencing spatial attention performance, such as handedness strength (Ochando and Zago, 2018), eye dominance is gaining increasing interest (e.g., Roth et al., 2002; Szczepanski and Kastner, 2013). Eye dominance and handedness have been shown to have a very weak relationship, with $66 \%$ of right-handers having a right DE and $60 \%$ of left-handers having a left DE (Bourassa et al., 1996). Numerous studies failed to show a clear link between the two, which is not surprising given the fact the afference/ efference of one hand is associate to one hemisphere whereas the efference of one eye is associated to both hemispheres (Mapp et al., 2003). The dominant eye is defined as the eye manifesting physiological superiority and it is the one chosen to align a target in peripersonal space with a more distant point, or when performing a monocular task such as looking through a small hole (it is referred to as sighting dominant eye; Porac and Coren, 1976). There are interocular differences in activation of visual areas in response to stimuli presented to the contralateral visual field, with the dominant eye generating a stronger activation in response to sensory information as compared to the one generated by the non-dominant one (Mendola and Conner, 2007; Rombouts et al., 1996). Since the dominant eye, as compared to the non-dominant one, activates larger portions of the ipsilateral primary visual cortex (Erdogan et al., 2002), it has been concluded that ocular dominance is mainly depending on the ipsilateral occipital lobe (Shima et al., 2010) possibly as a result of more numerous, or stronger inputs gathered by the temporal hemiretina and projecting to the ipsilateral visual cortex. Consistent with this last proposition, simple reaction times in response to a lateralized visual target are shorter for the contralateral visual hemifield with respect to the DE (Chaumillon et al., 2014). Globally, there is an advantage for stimuli processed by the dominant eye: perceived image size is increased (Coren and Porac, 1976) and percept is more salient, suggesting that information from the dominant eye has priority in visual processing (Shneor and Hochstein, 2008, 2006). Grounding on the activation-orientation theory (Reuter-Lorenz et al., 1990) we hypothesized that eye dominance may impact physiologically visuospatial attention bias, and namely that individuals with right dominant eye should show a greater amount of pseudoneglect as compared to individuals with a left dominant eye. The activationorientation theory, by postulating that the distribution of attention is biased toward the direction opposite to the most activated hemisphere, accounts for the pseudoneglect phenomenon as due to a biased perception of the half of the line in the left hemifield because of the right hemisphere dominance for visuospatial attention. We predicted that right-handed individuals with right dominant eye, as compared to righthanded individuals with left dominant eye, should show a greater amount of pseudoneglect. This would depend on of the fact that information from the left side of the line is perceived elongated because it is processed by the right hemisphere, which not only is the most activated by the attentional task, but also by the inputs of their right dominant eye to the right visual cortex (i.e., right hemisphere dominance plus dominant eye). By contrast, in individuals with left dominant eye, the leftward bias would be mitigated by the activation of the left hemisphere due to stronger inputs from the left dominant eye to the 
left visual cortex. We thus put forward here a simple model to predict the manifestation of pseudoneglect as a function of eye dominance: left eye dominance, by increasing the activity in the left visual cortex, would dampen the typical bias depending upon right hemisphere dominance. We tested this prediction by comparing the performance at both the perceptual (Landmark) and manual line bisection tasks, between one group of healthy right-handed participants with right dominant eye and one group of healthy right-handed participants with left dominant eye. These tasks, despite being both used to quantify visuospatial asymmetry (Zago et al., 2017), bear substantial differences (Milner et al., 1992). The Landmark task can be summarized as a pure test of perceptual/attentional bias, as it requires to judge a series of pre-bisected lines and, in our study, avoids any hand movement, whereas the motor line bisection task involves motor bias by requiring to actively bisect a line with the dominant hand. We therefore expect eye dominance and strength of handedness to have a different weight in the quantification of the visuospatial bias according to the task used to measure it. We predict eye dominance to manifest its influence on visuospatial bias when assessed in the more perceptual manner, i.e., the Landmark task, and handedness to show its influence on visuospatial bias when assessed by manual line bisection because of the motor bias intrinsic to the task. Our model and predictions were supported by the results, showing that eye dominance contributes in determining the presence and amount of pseudoneglect in the precited direction solely when assessed by the Landmark task. This finding may help account for the interindividual variability observed in the pseudoneglect phenomenon.

\section{Materials and methods}

\subsection{Participants}

Forty adults with normal or corrected-to-normal vision and no history of neurological problems were recruited for the study. All participants gave informed consent and were right-handed according to the Edinburgh Handedness Inventory, which is used to quantified the degree of handedness (Oldfield, 1971). The study was approved by the French national ethics committee (CPP SUD EST IV) and was conducted in accordance with the ethical standards of the 1964 Declaration of Helsinki. Twenty participants were in the right dominant eye (RDE) group and the other twenty in the left dominant eye (LDE) group. In order to have comparable numbers in the two groups, participants were screened for eye dominance by having them perform three repetitions of the hole-in-card test (Miles, 1930). The number of participants for each group was set to 20; once one group was complete recruitment continued for the other group. All participants had unambiguous eye dominance, as for all forty participants the hole in the card was aligned with the same eye on each repetition. Two participants were excluded because the Edinburgh Handedness Inventory score was missing. The final sample submitted to statistical analysis was composed by thirtyeight participants: eighteen in the RDE group (4 males, mean age $=26.4, \mathrm{SEM}=0.99, \mathrm{SD}=4.20$ ) and the remaining twenty in the LDE group (9 males, mean age $=28.4$ SEM $=1.18, \mathrm{SD}=5.25)$. The two groups did not differ in terms of age $[t(36)=-$ $1.257 p=0.217$ ], and similarly the degree of Handedness did not differ between the RDE (mean $=87.25$, SEM $=3.46$, SD $=$ 14.70) and LDE (mean $=80.06, \mathrm{SEM}=4.30, \mathrm{SD}=19.23$ ) group $[\mathrm{t}(36)=1.282 \mathrm{p}=0.208]$. Following the eye dominance test, participants performed both a perceptual (Landmark task; Milner et al., 1992) and manual (Schenkenberg et al., 1980) line bisection task, the order of which was counterbalanced across participants. During both tasks, which were carried out in binocular vision, participants were seated comfortably with their head positioned on a chinrest.

\subsection{Landmark task}

A modified version of the Landmark task (Milner et al., 1992) was used to quantify visuospatial performance. The Landmark task (as in Schintu et al., 2014) consisted of a series of pre-bisected lines presented centrally on a computer screen (22 inches (559 $\mathrm{mm} \times 274 \mathrm{~mm}$ ), resolution $1680 \times 1050$, refresh rate $60 \mathrm{HZ}$ ) positioned with the center aligned to the participant's mid-sagittal axis. Participants placed their hands on their lap beneath the table and were instructed to fully inspect each pre-bisected line and judge whether the mark (vertical transector) was closer to the left or right end of the line. In this two-alternative forcedchoice paradigm participants answered by pressing either the left (transector closer to the left end of the line) or right (transector closer to the right end of the line) pedal positioned under each foot. They were instructed to respond as accurately and quickly as possible. Prior to the task, at least ten practice trials were given to ensure that participants correctly understood the instructions and were confident answering with the pedals. The stimuli were white lines $(350 \mathrm{~mm} \times 2 \mathrm{~mm})$ displayed on a black screen positioned $35 \mathrm{~cm}$ from the participant's eyes. Lines were transected at the true center and at 2, 4, 6, 8, and $10 \mathrm{~mm}$ toward the left and right of the true center $(0 \mathrm{~mm})$. Each of the 11 different pre-bisected lines was presented six times in a random order, yielding a total of 66 trials. Each pre-bisected line was displayed for a maximum of $5 \mathrm{~s}$ or until a response was made and was then replaced by a black-and-white patterned mask which stayed on the screen for $1 \mathrm{~s}$ before the next prebisected line was displayed. Presentation software (Neurobehavioral Systems, Inc., USA) was used to generate the stimuli, record responses, and control the timing of stimulus presentation throughout the task. For each participant, the percentage of 'right' responses was plotted as a function of the position of the transector. These data were then fitted with a sigmoid function and the value on the $x$-axis corresponding to the point at which the participant responded 'right' $50 \%$ of the time was taken as that participant's point of subjective equality (PSE). This task prioritizes the perceptual and minimizes the motor component of the visuospatial bias by asking participants to judge a series or pre-bisected lines instead of actively bisect them (Milner et al., 1992).

\subsection{Manual line bisection task}

The manual line bisection task (Schenkenberg et al., 1980), consisted of a series of 10 black lines (350 $\mathrm{mm} \times \sim 2 \mathrm{~mm}$ which were of identical in size to those used for the Landmark task) each drawn on A3 (297 mm x $420 \mathrm{~mm}$ ) sheets of paper that were positioned over the computer screen which was kept at the same distance and position as for the Landmark task. Participants were instructed to fully inspect each line and with the pen held in their right hand, draw a vertical mark where they thought the center of the line to be. Once the mark had been drawn the experimenter then turned the page to reveal the following line. No time limit was imposed, and participants took on average $1 \mathrm{~s}$ to place the mark on each line. For each of the ten lines the distance between the mark placed by the participant and the true center of the line was calculated. The PSE was calculated as the average across the ten trials of the distance between the true center and the mark, with marks to the right of center coded as positive and to the left as negative. This task, while perceptual in nature, by asking participants to actively bisect a series of lines may nullify the perceptual bias because of the kind motor response involved (Milner et al., 1992).

\subsection{Statistical analyses}


Statistical analyses were carried out using the SPSS software package (Version 24, Chicago, IL: SPSS Inc.). The significance threshold was set at 0.05 (two-tailed). All values in the text are presented with standard error of the mean (SEM) and when appropriate also standard deviation (SD) and effect size are reported. The performances of the Landmark and Manual line bisection tasks were submitted to repeated measure Analysis of Variance (ANOVA) having Task (Manual line bisection, Landmark) as within variable, Group (RDE, LDE) as between variable and Degree of Handedness (i.e., Edinburgh score) as covariate. We included degree of handedness as covariate because it has been shown to modulate line bisection performance (Jewell and McCourt, 2000).

3. Results The repeated measure ANOVA revealed a main effect of Task $[F(1,35)=21.640, p<0.001, \eta 2 p=0.382]$ such that the PSE was more leftward (i.e., negative) in the Landmark task (mean $=1.02 \mathrm{~mm}$; SEM $=0.34$ ) than in the Manual line bisection task (mean $=0.32 \mathrm{~mm}$; SEM $=0.70$ ). The Task $x$ Group interaction $[F(1,35)=9.030, p=0.005, \eta 2 p=0.205]$ was significant, meaning that after we controlled for Degree of Handedness there was a significant difference in the performance between the RDE and LDE groups according to the task performed. The Task $x$ Degree of Handedness interaction was also significant $[F(1,35)=18.084, p<0.001, \eta 2 p=0.341]$ whereas the main effect of group was not $[F(1,35)=0.021, p=0.886$, $\eta 2 p=0.001]$. The subsequent independent univariate ANOVAs we ran to follow up the Task $x$ Group interaction revealed that, as quantified by the Landmark task, the RDE group had a greater amount of pseudoneglect (mean $=-1.85 \mathrm{~mm}, \mathrm{SEM}=0.46$ ) as compared to the LDE group (mean $=-0.28 \mathrm{SEM}=0.34)[\mathrm{F}(1,36)=6.083 p=0.019, \eta 2 p=0.145]$ (Fig. 1). In contrast, no difference was found between the RDE (mean $=0.81 \mathrm{SEM}=0.87$ ) and LDE (mean $=-0.12 \mathrm{SEM}=0.7$ ) groups at the manual line bisection task $[F(1,36)=0.423 p=0.520]$ (Fig. 1). Moreover, the amount of pseudoneglect as quantified by the Landmark task was significantly different from zero for the RDE group [t(17) $=-4.028 p=0.001]$, but not for the LDE group [t(19) $=-$ $0.635 p=0.533$ ] confirming the presence of pseudoneglect solely for the RDE group. To follow up the Task $x$ Degree of Handedness interaction we computed two Spearman correlations between the degree of handedness and the performance at each task. These analyses revealed a significant correlation between the degree of handedness and the performance solely for the Manual line bisection ( $r=-0.516 p=0.001)$, and not for the Landmark task ( $r=0.012 p=0.941)$ (Fig. 2). Based on (Ochando and Zago, 2018) finding, we categorized participants based on their degree of Handedness, independently of their eye dominance. Participants whose score at the Edinburgh task was $>85$ were categorize as "strong" righthanded $(n=21)$ and those whose score was $<85$ as "weak" right-handed $(n=17)$. The independent $t$-test comparing the motor line bisection performance revealed that the strong right-handed group showed a leftward bias (mean $=-1.23 \mathrm{SD}=4.20 \mathrm{SEM}=0.92$ ) and the weaker right-handed group showed a rightward bias (mean $=2.24 \mathrm{SD}=3.78 \mathrm{SEM}=0.92$ ) $[\mathrm{t}(36)=-2.645 \mathrm{p}=0.012$, Cohens $d=0.87]$, whereas no difference was found between the strong and weak handedness groups' performance at the Landmark task $[\mathrm{t}(36)=0.475 \mathrm{p}=0.638]$. These results show that in right handers, eye dominance did not affect performance on a manual line bisection task. Indeed, participants in both the RDE and LDE groups were very accurate when marking the center of the line with their right hand. In contrast, on the Landmark task the performance of the two groups differed significantly; the LDE group accurately judged the center close to the true center, whereas the RDE group exhibited a significant leftward bias as they judged the center to be significantly leftward of the true center.

4. Discussion We hypothesized that eye dominance impacts visuospatial attention, and thus that the asymmetric hemisphere activation over the visual areas triggered by eye dominance interacts with the typical right hemisphere activation in attentional task. The current findings support the hypothesis that eye dominance influences visuospatial attention mechanisms and, as predicted, shows that right-handed individuals with right dominant eye exhibit a greater amount of pseudoneglect compared to individuals with left eye dominance. The right dominant eye group showed a significant leftward bias and a greater amount of pseudoneglect than the group with left dominant eye only when measured by the Landmark task, but not by the manual line bisection task. The Landmark task (Milner et al., 1992) is typically administered to healthy participants whereas the manual line bisection (Schenkenberg et al., 1980) is the most common bedside tool for hemispatial neglect screening. These two tasks are implied to measure the same phenomenon and are anecdotally compared across populations, despite the effective correlation between them has never been produced and differences between the two have been reported in the literature. The difference in performance at the two tasks can lie in the amount of variability present in the manual line bisection performance and its poor sensitivity (McCourt and Jewell, 1999; McCourt and Olafson, 1997) as compared to the Landmark task. The Landmark task is thought to minimize the motor component and be more sensitive to the perceptual one (Milner et al., 1992), providing finer quantification of visuospatial attention bias than manual line bisection (Mclntosh et al., 2019). The greater amount of pseudoneglect observed with the Landmark task is the consequence of the elimination, minimization, and control of confounding factors such as the motor responses associated with the manual version of the line bisection (McCourt and Jewell, 1999; McCourt and Olafson, 1997). Our results, by showing that the influence of eye dominance was measurable only via the Landmark task (devoid of any hand movement) are coherent with such argument. The additional finding that the correlation between the degree of handedness and the amount of pseudoneglect was significant for the manual line bisection tasks, but not for the Landmark task, provides further support to this conclusion. Conclusion that is even further strengthened by the fact that, when participants were categorized and compared by handedness strength, difference in performance was observed for the manual line bisection and not for the Landmark task. Our model of eye-dominance dependent modulation of attentional biases was grounded on the activation-orientation theory (Reuter-Lorenz et al., 1990). This theory by postulating that the distribution of attention is biased to the direction opposite to the more activated hemisphere, offers an account for the pseudoneglect origin. Since visuospatial tasks such as line bisection involve activation of the right hemisphere (Fink et al., 2000) the contralateral (left) half of the line is the focus of greater attention and perceived as longer relative to the less attended right half, resulting in midpoint judgment located to the left of the veridical center (Bultitude and Aimola Davies, 2006). However, the biased perception of stimuli in the left hemifield, due to the increased activation of the right hemisphere in the attentional task, interacts with the priority in visual processing for stimuli processed by the dominant eye (Shneor and Hochstein, 2008, 2006) that is the result of the increased activation of the visual cortex ipsilateral the to the dominant eye (Shima et al., 2010). In participants with right dominant eye, the special relationship between the dominant eye and its ipsilateral hemisphere (i.e., the right hemisphere Shima et al., 2010) is consistent with the right asymmetry of the attentional networks. In contrast, in participants with left dominant eye, the privileged relationship between the dominant eye and its ipsilateral hemisphere (i.e., the left hemisphere) counteracts the right hemisphere dominance for visuospatial attention, possibly resulting in a smaller amount of pseudoneglect. The advocated involvement of primary visual areas in higher cognitive functions is not surprising since the traditional interpretation of the activity in the primary visual cortex has been challenged by findings showing that reward-timing activity can occur very early in sensory-processing paths such in the dominant eye visual area (Shuler and Bear, 2006). When considering the possible neural underpinning of the behavioral finding reported here, it is crucial to consider the key role of posterior parietal cortex (PPC) in the frontoparietal network, since its modulation by transcranial direct-current stimulation directly impacts the 
amount of pseudoneglect (i.e., in a Landmark task, Ribolsi et al., 2013), along with the specific relationship between the dominant eye and its ipsilateral hemisphere (Shima et al., 2010). The neural activity in the PPC is influenced by eye dominance. Indeed, an opposite asymmetry in interhemispheric transfer between right and left eyed participants has recently been demonstrated (Chaumillon et al., 2018). Based on this evidence we speculate that the dominant eye may influence visuospatial attention via the ipsilateral visual cortex triggering additional neural activation in the ipsilateral PPC, which would in turn affect the frontoparietal attentional network. Previous studies have indeed shown that the combination of eye dominance and handedness influences lateralization of the dorsal network (Petit et al., 2014), the asymmetry in the control of saccades amplitude and velocity (Vergilino-Perez et al., 2012), and impacts the neural mechanisms involved in converting visual input into motor commands (Chaumillon et al., 2014). However, this is the first study demonstrating the influence of sighting eye dominance on visuospatial attention in binocular vision. A current limitation of this study is that we selected right-handed participants. While it would be interesting to extend the investigation of the eye dominance effects to left-handed participants, this was done with the purpose of providing a first assessment of the effects of eye dominance in reference to the literature, and thus concerning the most representative part of the population, i.e., $90 \%$ of it is righthanders, for whom manual and attentional hemispheric dominance is relatively unambiguous. Indeed, pseudoneglect is generally observed at the population level, possibly due to the overrepresentation of right-handers with a right eye dominance in a randomly sampled population (Bourassa et al., 1996) which, according to our hypotheses, should show an attentional bias towards the left visual hemifield. The right-handers with a left dominant eye, on the other hand, could show a different attentional bias that would have been masked up to now by their under-representation in a randomly selected population. Indeed, when the population is examined according to eye dominance, the amount of pseudoneglect is reduced in the left and accentuated in the right dominant eye group. To conclude, this is the first study reporting the effect of eye dominance on visuospatial attention as measured by the Landmark task when performed binocularly, and thus suggest that asymmetric activation of primary visual areas may modulate visuospatial attention. By showing that motor line bisection performance is mainly related to the degree of handedness whereas the Landmark task's performance to eye dominance, these results have implication in application of those visuospatial tasks that, contrary to what anecdotally reported, are not assessing the same phenomenon and as such should not be interchanged. Finally, these findings not only point at the role of eye dominance as a variable in spatial attention but help in better defining intact visuospatial cognition mechanism and, ultimately encouraging further research to pinpoint the neural basis of such phenomenon.

\section{CRediT authorship contribution statement}

S. Schintu: Conceptualization, Data curation, Formal analysis, Writing - original draft. R. Chaumillon: Conceptualization, Data curation, Formal analysis. A. Guillaume: Conceptualization, Writing - review \& editing. R. Salemme: Software. K.T. Reilly: Writing - original draft. L. Pisella: Conceptualization, Writing - review \& editing. A. Farnè: Conceptualization, Writing - original draft, Writing - review \& editing.

\section{Acknowledgments}

This work was supported by ANR-11-LABEX-0042, the Fondation pour la Recherche Méedicale, the Fondation de France, Neuro-Ophtalmology Berthe Fouassier Scholarship (2011-00020576, 2012-00031565 2018 to AF), the Société Française d'Ophtalmologie and a James S. McDonnell Foundation Scholar Award (to AF), and the National Institutes of Health Ruth L. Kirschstein National Research Service Award (to SS).

\section{References}

Bourassa, D.C., McManus, I.C., Bryden, M.P., 1996. Handedness and eye-dominance: a meta-analysis of their relationship. Laterality $1,5-34$. https://doi.org/10.1080/ 713754206.

Bowers, D., Heilman, K.M., 1980. Pseudoneglect: effects of hemispace on a tactile line bisection task. Neuropsychologia 18, 491-498.

Bultitude, J.H., Aimola Davies, A.M., 2006. Putting attention on the line: investigating the activation-orientation hypothesis of pseudoneglect. Neuropsychologia 44 , 1849-1858. https://doi.org/10.1016/j. neuropsychologia.2006.03.001. Chaumillon, R., Blouin, J., Guillaume, A., 2018. Interhemispheric transfer time asymmetry of visual information depends on eye dominance: an electrophysiological study. Front. Neurosci. 12 https://doi.org/10.3389/fnins.2018.00072.

Chaumillon, R., Blouin, J., Guillaume, A., 2014. Eye dominance influences triggering action: the Poffenberger paradigm revisited. Cortex 58, 86-98. https://doi.org/ 10.1016/j.cortex.2014.05.009.

Coren, S., Porac, C., 1976. Size accentuation in the dominant eye. Nature 260, 527-528. Erdogan, A.R., €Ozdikici, M., Aydin, M.D., Aktas, €O., Dane, S., 2002. Right and left visual cortex areas in healthy subjects with right- and left-eye dominance. Int. J. Neurosci. 112, 517-523. https://doi.org/10.1080/00207450290025626.

Fink, G.R., Marshall, J.C., Shah, N.J., Weiss, P.H., Halligan, P.W., Grosse-Ruyken, M., Ziemons, K., Zilles, K., Freund, H.J., 2000. Line bisection judgments implicate right par in Jewell, G., McCourt, M.E., 2000. Pseudoneglect: a review and meta-analysis of Jewell, G., McCourt, M.E., 2000. Pseudoneglect: a review and meta-analysis of
performance factors in line bisection tasks. Neuropsychologia 38, 93-110. https:/ doi.org/10.1016/S0028-3932(99)00045-7.

Kinsbourne, M., 1970. A model for the mechanism of unilateral neglect of space. Trans. Am. Neurol. Assoc. 95, 143-146.

Manning, L., Halligan, P.W., Marshall, J.C., 1990. Individual variation in line bisection: a study of normal subjects with application to the interpretation of visual neglect.

Neuropsychologia 28, 647-655.

Mapp, A.P., Ono, H., Barbeito, R., 2003. What does the dominant eye dominate? A brie and somewhat contentious review. Percept. Psychophys. 65, 310-317.

McCourt, M.E., Jewell, G., 1999. Visuospatial attention in line bisection: stimulus

modulation of pseudoneglect. Neuropsychologia 37, 843-855.

McCourt, M.E., Olafson, C., 1997. Cognitive and perceptual influences on visual line

bisection: psychophysical and chronometric analyses of pseudoneglect.

Neuropsychologia 35, 369-380.

McIntosh, R.D., Brown, B.M.A., Young, L., 2019. Meta-analysis of the visuospatial aftereffects of prism adaptation, with two novel experiments. Cortex 111, 256-273. https://doi.org/10.1016/j.cortex.2018.11.013.

Mendola, J.D., Conner, I.P., 2007. Eye dominance predicts fMRI signals in human retinotopic cortex. Neurosci. Lett. 414, 30-34. https://doi.org/10.1016/j.

neulet.2006.12.012.

Mesulam, M.M., 1999. Spatial attention and neglect: parietal, frontal and cingulate 
contributions to the mental representation and attentional targeting of salient extrapersonal events. Philos. Trans. R. Soc. Lond. B. Biol. Sci. 354, 1325-1346. https://doi.org/10.1098/rstb.1999.0482.

Milner, A.D., Brechmann, M., Pagliarini, L., 1992. To halve and to halve not: an analysis

of line bisection judgements in normal subjects. Neuropsychologia 30, 515-526.

Nicholls, M.E., Roberts, G.R., 2002. Can free-viewing perceptual asymmetries be explained by scanning, pre-motor or attentional biases? Cortex 38, 113-136.

https://doi.org/10.1016/S0010-9452(08)70645-2.

Ochando, A., Zago, L., 2018. What are the contributions of handedness, sighting dominance, hand used to bisect, and visuospatial line processing to the behavioral line bisection bias? Front. Psychol. 9 https://doi.org/10.3389/fpsyg.2018.01688. Oldfield, R.C., 1971. The assessment and analysis of handedness: the Edinburgh inventory. Neuropsychologia 9, 97-113. https://doi.org/10.1016/0028-3932(71) 90067-4.

Petit, L., Zago, L., Mellet, E., Jobard, G., Crivello, F., Joliot, M., Mazoyer, B., TzourioMazoyer, N., 2014. Strong rightward lateralization of the dorsal attentional network in left-handers with right sighting-eye: an evolutionary advantage. Hum. Brain Mapp. https://doi.org/10.1002/hbm.22693 n/a-n/a.

Porac, C. Coren, S., 1976. The dominant eye. Psychol. Bull. 83, 880-897.

Ribolsi, M., Lisi, G., Lorenzo, G.D., Koch, G., Oliveri, M., Magni, V., Pezzarossa, B Saya, A., Rociola, G., Rubino, I.A., Niolu, C., Siracusano, A., 2013. Perceptual pseudoneglect in schizophrenia: candidate endophenotype and the role of the right parietal cortex. Schizophr. Bull. 39, 601-607. https://doi.org/10.1093/schbul/ sbs036.

Rombouts, S.A.R.B., Barkhof, F., Sprenger, M., Valk, Jaap, Scheltens, P., 1996. The functional basis of ocular dominance: functional MRI (fMRI) findings. Neurosci. Lett. 221, 1-4. https://doi.org/10.1016/S0304-3940(96)13260-2.

Roth, H.L., Lora, A.N., Heilman, K.M., 2002. Effects of monocular viewing and eye dominance on spatial attention. Brain 125, 2023-2035. https://doi.org/10.1093/ brain/awf210.

Schenkenberg, T., Bradford, D.C., Ajax, E.T., 1980. Line bisection and unilateral visual neglect in patients with neurologic impairment. Neurology 30, 509-517.

Schintu, S., Pisella, L., Jacobs, S., Salemme, R., Reilly, K.T., Farn e, A., 2014. Prism adaptation in the healthy brain: the shift in line bisection judgments is long lasting and fluctuates. Neuropsychologia 53, 165-170. https://doi.org/10.1016/].

neuropsychologia.2013.11.013.

Shima, H., Hasegawa, M., Tachibana, O., Nomura, M., Yamashita, J., Ozaki, Y., Kawai, J.,

Higuchi, M., Kado, H., 2010. Ocular dominance affects magnitude of dipole moment:

an MEG study. Neuroreport 21, 817-821. https://doi.org/10.1097/

WNR.0b013e32833ce5d7. 


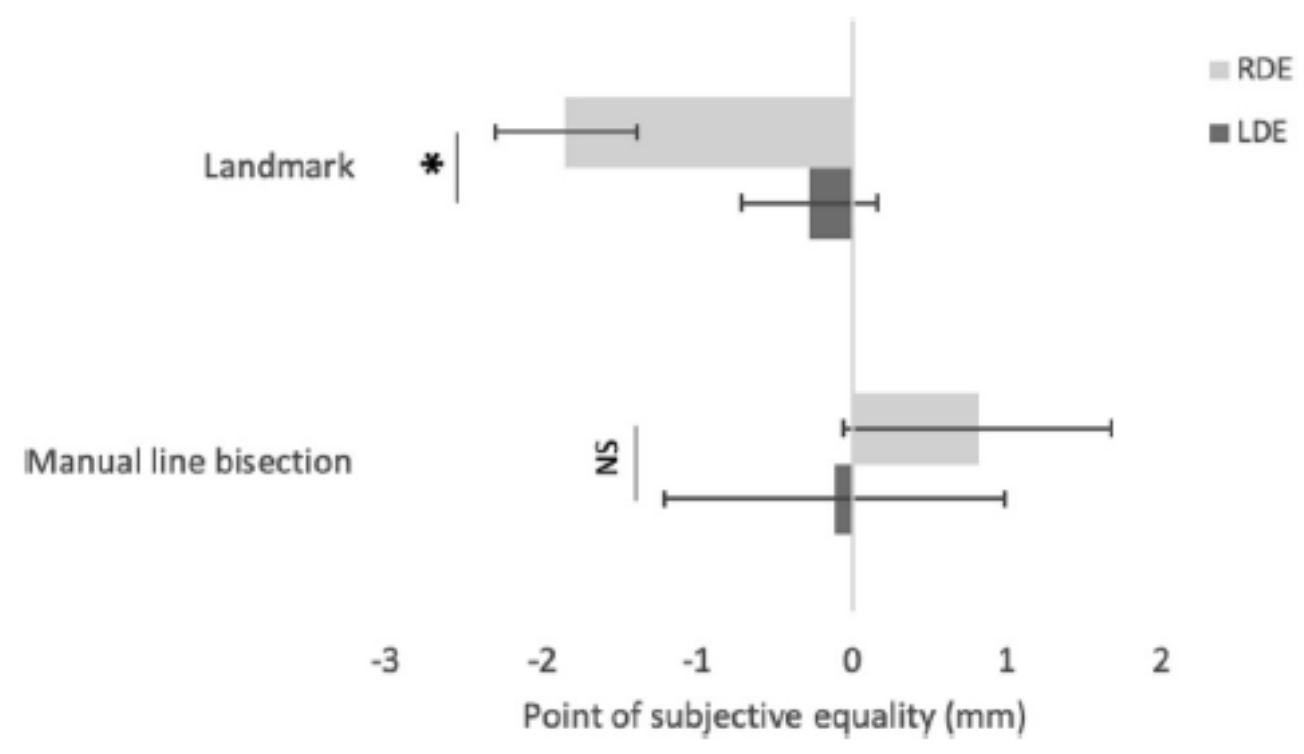

Fig. 1. Point of Subjective Equality (PSE) at the Landmark and Manual line bisection tasks as a function of eye dominance. Negative values represent a PSE to the left of the true center (pseudoneglect). Values are means and error bars represent the SEM.
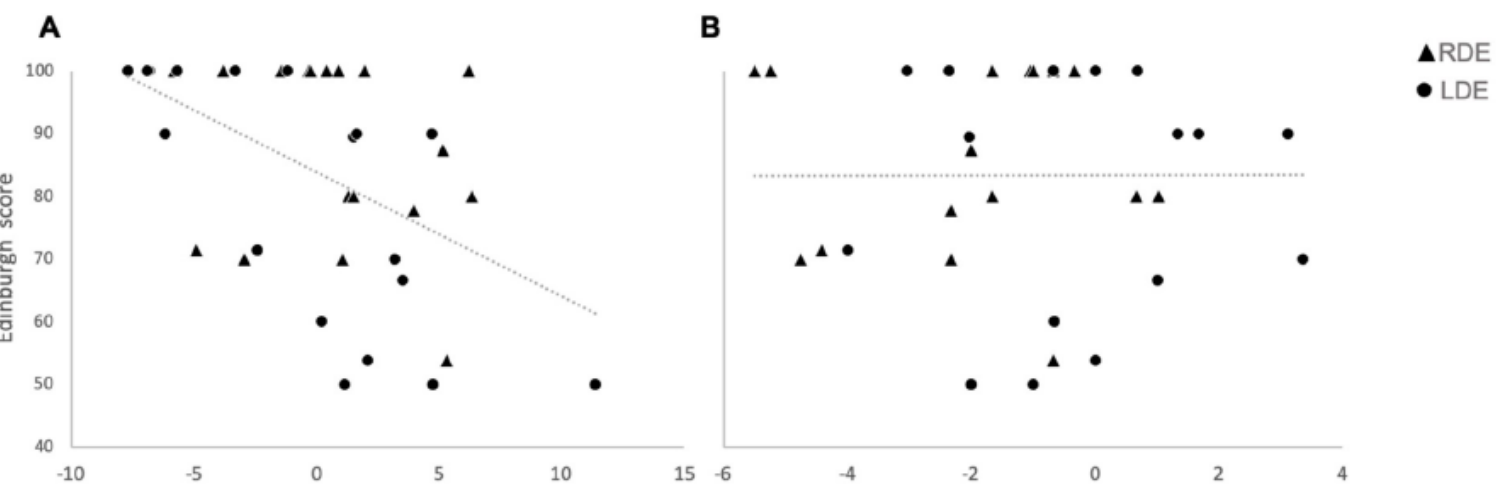

Point of subjective equality $(\mathrm{mm})$

Fig. 2. Pearson correlation between Edinburgh scale score and manual line bisection task (panel A) and Landmark task (panel B). 